\title{
Prediction of Partition Coefficients of Organic Compounds for SPME/PDMS
}

\author{
Hsuan-Yu LIAO ${ }^{1}$, Miao-Ling HUANG ${ }^{2}$, Yu-Ting $\mathrm{LU}^{1}$ and Keh-Ping CHAO ${ }^{1, *}$ \\ ${ }^{1}$ Department of Occupational Safety and Health, China Medical University, 91 Hsueh-Shih Rd., \\ Taichung 40402, Taiwan \\ ${ }^{2}$ Department of Industrial Management, ChienHsin University of Science \& Technology, 229 \\ Jianxing Rd., Taoyuan 32097, Taiwan
}

\begin{abstract}
The partition coefficients of 51 organic compounds between SPME/PDMS and gas were compiled from the literature sources in this study. The effect of physicochemical properties and descriptors on the partitioning process of partition coefficients was explicated by the correlation analysis. The PDMS-gas partition coefficients were well correlated to the molecular weight of organic compounds $(r=0.832, p<0.05)$. An empirical model, consisting of the molecular weight and the polarizability, was developed to appropriately predict the partition coefficients of organic compounds. The empirical model for estimating the PDMS-gas partition coefficient will contribute to the practical applications of the SPME technique.
\end{abstract}

\section{Introduction}

Solid phase microextraction (SPME) is a solvent-free sample preparation technique. Polymeric materials, such as polydimethylsiloixane (PDMS), are coated onto the outer layer of the fused silica rod of the SPME device. According to the principle of partitioning, the analyte is adsorbed from the sample matrix onto the coating stationary phase. The concentrated extract is then transferred to an instrument, such as gas chromatography, for thermal desorption and analysis. Prior to using the SPME technique for sampling and analysis, the first step is to select the appropriate polymer as the SPME fiber and to determine the equilibrium extraction time for SPME, which is primarily based on the partition

\footnotetext{
* Corresponding author:kpchao@mail.cmu.edu.tw
} 
coefficient of the analyte between the SPME fiber and the sample matrix.

The objective of this study is to develop predictive equations for the partition coefficients of a variety of organic compounds in gas onto SPME/PDMS. This was achieved by using the PDMS-gas partition coefficients retrieved from the literature and relating them to their physicochemical properties and molecular descriptors, such as molecular connectivity index $\left({ }^{1} \chi\right)$, water solubility (WS), molecular weight $(M \mathrm{~W})$, octanol-water partition coefficient $\left(\log K_{\text {ow }}\right)$, and polarizability $(\Phi)$. The results of this study can facilitate the application of the SPME technique.

\section{Method}

In this study, the PDMS-gas partition coefficients $K_{f g}$ for alkanes and aromatic hydrocarbons was compiled from the literature published by Dr. Pawliszyn [1]. As shown in Table 1, the values of $K_{f g}(n=51)$ exhibit a wide range from 157 (for benzene) to 95,700 (for 3,3-dimethyloctane).

Table 1the Training Set Of Pdms-Gas Partition Coefficients And Physico-Chemical Descriptors

\begin{tabular}{|c|c|c|c|c|c|c|c|}
\hline Compound & $K_{f g}$ & ${ }^{1} \chi$ & $\Phi$ & $I$ & $\begin{array}{c}M_{\mathrm{W}}(\mathrm{g} / \mathrm{mol} \\
\mathrm{e}) \\
\end{array}$ & $\begin{array}{c}W S \\
(\mathrm{mg} / \mathrm{L}) \\
\end{array}$ & $\begin{array}{l}\log \\
K_{\mathrm{ow}}\end{array}$ \\
\hline 3-Methylpentane & 159 & $\begin{array}{c}2.80 \\
8 \\
\end{array}$ & $\begin{array}{l}1.7 \\
82 \\
\end{array}$ & 0 & 86.18 & 17.9 & 3.6 \\
\hline 2,4-Dimethylpentane & 262 & $\begin{array}{c}3.12 \\
6\end{array}$ & $\begin{array}{r}2.1 \\
19\end{array}$ & 0 & 100.2 & 5.5 & 3.63 \\
\hline 2,2,3-Trimethylpentane & 569 & $\begin{array}{l}3.48 \\
14\end{array}$ & $\begin{array}{l}2.4 \\
56\end{array}$ & 0 & 114.20 & 2.40 & 4.09 \\
\hline 2,3-Dimethylhexane & 968 & $\begin{array}{l}3.68 \\
08\end{array}$ & $\begin{array}{l}2.4 \\
56\end{array}$ & 0 & 114.23 & 9.20 & 4.12 \\
\hline 2-Methylheptane & 993 & $\begin{array}{l}3.77 \\
01 \\
\end{array}$ & $\begin{array}{l}2.4 \\
56\end{array}$ & 0 & 114.23 & 7.97 & 4.20 \\
\hline 4-Methylheptane & $\begin{array}{l}1,06 \\
0\end{array}$ & $\begin{array}{l}3.80 \\
80\end{array}$ & $\begin{array}{l}2.4 \\
56\end{array}$ & 0 & 114.23 & 7.97 & 4.20 \\
\hline 3-Methylheptane & $\begin{array}{l}1,09 \\
0\end{array}$ & $\begin{array}{l}3.80 \\
80 \\
\end{array}$ & $\begin{array}{l}2.4 \\
56 \\
\end{array}$ & 0 & 114.23 & 0.792 & 4.20 \\
\hline 3-Ethylhexane & 990 & $\begin{array}{l}3.84 \\
59 \\
\end{array}$ & $\begin{array}{l}2.4 \\
56 \\
\end{array}$ & 0 & 114.23 & 7.97 & 4.20 \\
\hline 2,5-Dimethylheptane & $\begin{array}{l}1,97 \\
0\end{array}$ & $\begin{array}{l}4.16 \\
39 \\
\end{array}$ & $\begin{array}{l}2.7 \\
93 \\
\end{array}$ & 0 & 126.28 & 3.11 & 4.61 \\
\hline 3,5-Dimethylheptane & $\begin{array}{l}1,96 \\
0\end{array}$ & $\begin{array}{l}4.20 \\
18\end{array}$ & $\begin{array}{l}2.7 \\
93 \\
\end{array}$ & 0 & 128.26 & 3.11 & 4.61 \\
\hline 3,3-Dimethylheptane & $\begin{array}{l}2,09 \\
0\end{array}$ & $\begin{array}{l}4.12 \\
14 \\
\end{array}$ & $\begin{array}{l}2.7 \\
93 \\
\end{array}$ & 0 & 128.26 & 2.90 & 4.65 \\
\hline
\end{tabular}




\begin{tabular}{|c|c|c|c|c|c|c|c|}
\hline 2,3-Dimethylheptane & $\begin{array}{l}2,39 \\
0\end{array}$ & $\begin{array}{l}4.18 \\
08\end{array}$ & $\begin{array}{l}2.7 \\
93\end{array}$ & 0 & 128.26 & 3.37 & 4.61 \\
\hline 3,4-Dimethylheptane & $\begin{array}{l}2,42 \\
0\end{array}$ & $\begin{array}{l}4.21 \\
87\end{array}$ & $\begin{array}{l}2.7 \\
93\end{array}$ & 0 & 128.26 & 3.11 & 4.61 \\
\hline 2-Methyloctane & $\begin{array}{l}2,60 \\
0\end{array}$ & $\begin{array}{l}4.27 \\
01\end{array}$ & $\begin{array}{l}2.7 \\
93\end{array}$ & 0 & 128.26 & 2.87 & 4.69 \\
\hline 3-Methyloctane & $\begin{array}{l}2,89 \\
0\end{array}$ & $\begin{array}{l}4.30 \\
80\end{array}$ & $\begin{array}{l}2.7 \\
93\end{array}$ & 0 & 128.26 & 2.87 & 4.69 \\
\hline 3,3-Diethylpentane & $\begin{array}{l}2,61 \\
0 \\
\end{array}$ & $\begin{array}{l}4.24 \\
28\end{array}$ & $\begin{array}{l}2.7 \\
93 \\
\end{array}$ & 0 & 128.00 & 2.90 & 4.65 \\
\hline 2,2-Dimethylpentane & $\begin{array}{l}4,32 \\
0\end{array}$ & $\begin{array}{l}3.06 \\
07\end{array}$ & $\begin{array}{l}2.1 \\
19\end{array}$ & 0 & 100.20 & 4.40 & 3.67 \\
\hline 3,3-Dimethylpentane & $\begin{array}{l}5,05 \\
0\end{array}$ & $\begin{array}{l}3.12 \\
14\end{array}$ & $\begin{array}{l}2.1 \\
19\end{array}$ & 0 & 100.20 & 5.92 & 3.67 \\
\hline 2,3-Dimethylpentane & $\begin{array}{l}6,10 \\
0\end{array}$ & $\begin{array}{l}3.18 \\
08\end{array}$ & $\begin{array}{l}2.1 \\
19 \\
\end{array}$ & 0 & 100.20 & 5.25 & 3.63 \\
\hline 2-Methylnonane & $\begin{array}{l}6,69 \\
0\end{array}$ & $\begin{array}{l}4.77 \\
01\end{array}$ & $\begin{array}{l}3.1 \\
30\end{array}$ & 0 & 142.28 & 0.899 & 5.18 \\
\hline 3-Methylnonane & $\begin{array}{l}7,10 \\
0\end{array}$ & $\begin{array}{l}4.80 \\
80\end{array}$ & $\begin{array}{l}3.1 \\
30\end{array}$ & 0 & 142.28 & 0.97 & 5.18 \\
\hline Benzene & 301 & $\begin{array}{l}1.99 \\
98\end{array}$ & $\begin{array}{l}0.1 \\
65\end{array}$ & 1 & 78.11 & 1790.0 & $\begin{array}{l}0.21 \\
3\end{array}$ \\
\hline Toluene & 818 & $\begin{array}{l}2.41 \\
06\end{array}$ & $\begin{array}{l}0.5 \\
02\end{array}$ & 1 & 92.14 & 526.00 & 2.73 \\
\hline Ethylbenzene & $\begin{array}{l}2,07 \\
0\end{array}$ & $\begin{array}{l}2.97 \\
13\end{array}$ & $\begin{array}{l}0.8 \\
39\end{array}$ & 1 & 106.17 & 169.00 & 3.15 \\
\hline m-Xylene & $\begin{array}{l}2,09 \\
0 \\
\end{array}$ & $\begin{array}{l}2.82 \\
14 \\
\end{array}$ & $\begin{array}{l}0.8 \\
39 \\
\end{array}$ & 1 & 106.00 & 161.00 & 3.20 \\
\hline p-Xylene & $\begin{array}{l}2,50 \\
0\end{array}$ & $\begin{array}{l}2.82 \\
14\end{array}$ & $\begin{array}{l}0.8 \\
39\end{array}$ & 1 & 106.16 & 162.00 & 3.15 \\
\hline o-Xylene & $\begin{array}{l}2,90 \\
0\end{array}$ & $\begin{array}{l}2.82 \\
73\end{array}$ & $\begin{array}{l}0.8 \\
39\end{array}$ & 1 & 106.00 & 178.00 & 3.12 \\
\hline Isopropylbenzene & $\begin{array}{l}3,88 \\
0\end{array}$ & $\begin{array}{l}3.35 \\
41\end{array}$ & $\begin{array}{l}1.1 \\
76\end{array}$ & 1 & 120.19 & 61.30 & 3.66 \\
\hline n-Propylbenzene & $\begin{array}{l}5,04 \\
0\end{array}$ & $\begin{array}{l}3.47 \\
13\end{array}$ & $\begin{array}{l}1.1 \\
76 \\
\end{array}$ & 1 & 120.19 & 52.20 & 3.69 \\
\hline $\begin{array}{l}\text { 1-Methyl-3-Ethylbenze } \\
\text { ne }\end{array}$ & $\begin{array}{l}4,75 \\
0\end{array}$ & $\begin{array}{l}3.38 \\
21\end{array}$ & $\begin{array}{l}1.1 \\
76\end{array}$ & 1 & 120.19 & 40.00 & 3.98 \\
\hline $\begin{array}{l}\text { 1-Methyl-4-Ethylbenze } \\
\text { ne }\end{array}$ & $\begin{array}{l}6,23 \\
0\end{array}$ & $\begin{array}{l}3.38 \\
21\end{array}$ & $\begin{array}{l}1.1 \\
76\end{array}$ & 1 & 120.19 & 94.90 & 3.63 \\
\hline 1,3,5-Trimethylbenzene & $\begin{array}{l}6,48 \\
0 \\
\end{array}$ & $\begin{array}{l}3.23 \\
22 \\
\end{array}$ & $\begin{array}{l}1.1 \\
76 \\
\end{array}$ & 1 & 120.19 & 48.20 & 3.42 \\
\hline Isobutylbenzene & $\begin{array}{l}8,36 \\
0\end{array}$ & $\begin{array}{l}3.82 \\
72\end{array}$ & $\begin{array}{l}1.5 \\
13\end{array}$ & 1 & 134.22 & 10.10 & 4.68 \\
\hline sec-Butylbenzene & $\begin{array}{l}8,59 \\
0\end{array}$ & $\begin{array}{l}3.89 \\
20\end{array}$ & $\begin{array}{l}1.5 \\
13\end{array}$ & 1 & 134.22 & 17.60 & 4.57 \\
\hline $\begin{array}{l}\text { 1-Methyl-3-Isopropylb } \\
\text { enzene }\end{array}$ & $\begin{array}{l}10,1 \\
00\end{array}$ & $\begin{array}{l}3.76 \\
49 \\
\end{array}$ & $\begin{array}{l}1.5 \\
13 \\
\end{array}$ & 1 & 134.22 & 42.50 & 4.50 \\
\hline $\begin{array}{l}\text { 1-Methyl-4-Isopropylb } \\
\text { enzene }\end{array}$ & $\begin{array}{l}10,2 \\
00\end{array}$ & $\begin{array}{l}3.76 \\
49\end{array}$ & $\begin{array}{l}1.5 \\
13\end{array}$ & 1 & 134.22 & 23.40 & 4.10 \\
\hline
\end{tabular}




\begin{tabular}{|c|c|c|c|c|c|c|c|}
\hline $\begin{array}{l}\text { 1-Methyl-3-n-Propylbe } \\
\text { nzene }\end{array}$ & $\begin{array}{l}13,2 \\
00\end{array}$ & $\begin{array}{l}3.88 \\
21\end{array}$ & $\begin{array}{l}1.5 \\
13 \\
\end{array}$ & 1 & 134.22 & 9.09 & 4.67 \\
\hline $\begin{array}{l}\text { 1,2-Dimethyl-4-Ethylbe } \\
\text { nzene }\end{array}$ & $\begin{array}{l}17,4 \\
00\end{array}$ & $\begin{array}{l}3.79 \\
88\end{array}$ & $\begin{array}{l}1.5 \\
13\end{array}$ & 1 & 134.22 & 12.70 & 4.50 \\
\hline $\begin{array}{l}\text { 1,3-Dimethyl-2-Ethylbe } \\
\text { nzene }\end{array}$ & $\begin{array}{l}18,1 \\
00\end{array}$ & $\begin{array}{l}3.80 \\
47\end{array}$ & $\begin{array}{l}1.5 \\
13\end{array}$ & 1 & 134.22 & 19.60 & 4.28 \\
\hline $\begin{array}{l}\text { 1,2-Dimethyl-3-Ethylbe } \\
\text { nzene }\end{array}$ & $\begin{array}{l}20,0 \\
00\end{array}$ & $\begin{array}{l}3.80 \\
47\end{array}$ & $\begin{array}{l}1.5 \\
13\end{array}$ & 1 & 134.22 & 17.40 & 4.34 \\
\hline $\begin{array}{l}\text { 1,2,4,5-Tetramethylben } \\
\text { zene }\end{array}$ & $\begin{array}{l}24,7 \\
00\end{array}$ & $\begin{array}{l}3.65 \\
48\end{array}$ & $\begin{array}{l}1.5 \\
13\end{array}$ & 1 & 134.22 & 3.48 & 4.00 \\
\hline 2-Methylbutylbenzene & $\begin{array}{l}24,1 \\
00\end{array}$ & $\begin{array}{l}3.78 \\
77\end{array}$ & $\begin{array}{l}1.8 \\
50\end{array}$ & 1 & 148.24 & 12.70 & 4.43 \\
\hline n-Pentylbenzene & $\begin{array}{l}34,5 \\
00\end{array}$ & $\begin{array}{l}4.47 \\
13\end{array}$ & $\begin{array}{l}1.8 \\
50\end{array}$ & 1 & 148.24 & 3.37 & 4.90 \\
\hline $1,3,5$-Triethylbenzene & $\begin{array}{l}67,3 \\
00\end{array}$ & $\begin{array}{l}4.91 \\
43\end{array}$ & $\begin{array}{l}2.1 \\
87\end{array}$ & 1 & 162.27 & 0.014 & 5.11 \\
\hline $1,2,4$-Triethylbenzene & $\begin{array}{l}75,6 \\
00\end{array}$ & $\begin{array}{l}4.92 \\
02\end{array}$ & $\begin{array}{l}2.1 \\
87\end{array}$ & 1 & 162.27 & 2.90 & 5.11 \\
\hline n-Hexylbenzene & $\begin{array}{l}90,1 \\
00\end{array}$ & $\begin{array}{l}4.97 \\
13\end{array}$ & $\begin{array}{l}2.1 \\
87 \\
\end{array}$ & 1 & 162.20 & 0.902 & 5.52 \\
\hline $\begin{array}{l}\text { 1-Methyl-3-Isopropylb } \\
\text { enzene }\end{array}$ & $\begin{array}{l}10,1 \\
00\end{array}$ & $\begin{array}{l}3.76 \\
49\end{array}$ & $\begin{array}{l}1.5 \\
13\end{array}$ & 1 & 134.22 & 42.50 & 4.50 \\
\hline $\begin{array}{l}\text { 1-Methyl-4-Isopropylb } \\
\text { enzene }\end{array}$ & $\begin{array}{l}10,2 \\
00\end{array}$ & $\begin{array}{l}3.76 \\
49\end{array}$ & $\begin{array}{l}1.5 \\
13\end{array}$ & 1 & 134.22 & 23.40 & 4.10 \\
\hline $\begin{array}{l}\text { 1-Methyl-3-n-Propylbe } \\
\text { nzene }\end{array}$ & $\begin{array}{l}13,2 \\
00\end{array}$ & $\begin{array}{l}3.88 \\
21 \\
\end{array}$ & $\begin{array}{l}1.5 \\
13 \\
\end{array}$ & 1 & 134.22 & 9.09 & 4.67 \\
\hline $\begin{array}{l}\text { 1,2-Dimethyl-4-Ethylbe } \\
\text { nzene }\end{array}$ & $\begin{array}{l}17,4 \\
00\end{array}$ & $\begin{array}{l}3.79 \\
88\end{array}$ & $\begin{array}{l}1.5 \\
13\end{array}$ & 1 & 134.22 & 12.70 & 4.50 \\
\hline $\begin{array}{l}\text { 1,3-Dimethyl-2-Ethylbe } \\
\text { nzene }\end{array}$ & $\begin{array}{l}18,1 \\
00\end{array}$ & $\begin{array}{l}3.80 \\
47\end{array}$ & $\begin{array}{l}1.5 \\
13\end{array}$ & 1 & 134.22 & 19.60 & 4.28 \\
\hline $\begin{array}{l}\text { 1,2-Dimethyl-3-Ethylbe } \\
\text { nzene }\end{array}$ & $\begin{array}{l}20,0 \\
00\end{array}$ & $\begin{array}{l}3.80 \\
47\end{array}$ & $\begin{array}{l}1.5 \\
13 \\
\end{array}$ & 1 & 134.22 & 17.40 & 4.34 \\
\hline $\begin{array}{l}\text { 1,2,4,5-Tetramethylben } \\
\text { zene }\end{array}$ & $\begin{array}{l}24,7 \\
00\end{array}$ & $\begin{array}{l}3.65 \\
48\end{array}$ & $\begin{array}{l}1.5 \\
13\end{array}$ & 1 & 134.22 & 3.48 & 4.00 \\
\hline 2-Methylbutylbenzene & $\begin{array}{l}24,1 \\
00\end{array}$ & $\begin{array}{l}3.78 \\
77\end{array}$ & $\begin{array}{l}1.8 \\
50\end{array}$ & 1 & 148.24 & 12.70 & 4.43 \\
\hline n-Pentylbenzene & $\begin{array}{l}34,5 \\
00 \\
\end{array}$ & $\begin{array}{l}4.47 \\
13 \\
\end{array}$ & $\begin{array}{l}1.8 \\
50\end{array}$ & 1 & 148.24 & 3.37 & 4.90 \\
\hline 1,3,5-Triethylbenzene & $\begin{array}{l}67,3 \\
00\end{array}$ & $\begin{array}{l}4.91 \\
43\end{array}$ & $\begin{array}{l}2.1 \\
87\end{array}$ & 1 & 162.27 & 0.014 & 5.11 \\
\hline 1,2,4-Triethylbenzene & $\begin{array}{l}75,6 \\
00\end{array}$ & $\begin{array}{l}4.92 \\
02\end{array}$ & $\begin{array}{l}2.1 \\
87 \\
\end{array}$ & 1 & 162.27 & 2.90 & 5.11 \\
\hline n-Hexylbenzene & $\begin{array}{l}90,1 \\
00\end{array}$ & $\begin{array}{l}4.97 \\
13\end{array}$ & $\begin{array}{l}2.1 \\
87\end{array}$ & 1 & 162.20 & 0.902 & 5.52 \\
\hline $\begin{array}{l}\text { 1-Methyl-3-Isopropylb } \\
\text { enzene }\end{array}$ & $\begin{array}{l}10,1 \\
00\end{array}$ & $\begin{array}{l}3.76 \\
49\end{array}$ & $\begin{array}{l}1.5 \\
13\end{array}$ & 1 & 134.22 & 42.50 & 4.50 \\
\hline $\begin{array}{l}\text { 1-Methyl-4-Isopropylb } \\
\text { enzene }\end{array}$ & $\begin{array}{l}10,2 \\
00\end{array}$ & $\begin{array}{l}3.76 \\
49 \\
\end{array}$ & $\begin{array}{l}1.5 \\
13 \\
\end{array}$ & 1 & 134.22 & 23.40 & 4.10 \\
\hline $\begin{array}{l}\text { 1-Methyl-3-n-Propylbe } \\
\text { nzene }\end{array}$ & $\begin{array}{l}13,2 \\
00\end{array}$ & $\begin{array}{l}3.88 \\
21\end{array}$ & $\begin{array}{l}1.5 \\
13 \\
\end{array}$ & 1 & 134.22 & 9.09 & 4.67 \\
\hline
\end{tabular}


The polarizability and molecular connectivity index were used to represent the interactions between the analyte and PDMS coating. As shown in Eq. (1), the polarizability $\Phi$ was determined by the addition of atom/bond contribution factors $[2,3]$.

$$
\Phi=\sum F_{i} \times(\text { number of atom } / \text { bond })
$$

where $F_{i}$ is the contribution factors indicated in Table 2 .

Table 2. Contribution factors for the calculation of polarizability.

\begin{tabular}{|c|c|c|c|}
\hline Atom/bond & Contribution factor & Atom/bond & Contribution factor \\
\hline Carbon & 0.577 & Iodine & 0.407 \\
\hline Hydrogen* & -0.120 & Fluorine & -0.570 \\
\hline Oxygen & -0.825 & Cycle & -0.952 \\
\hline Hydroxyl & -3.701 & Double bond & -0.859 \\
\hline Chlorine & -0.187 & Triple bond & -0.109 \\
\hline Bromine & -0.222 & - & - \\
\hline
\end{tabular}

Data was obtained from Nirmalakhandan and Speece [2]; *Attached to carbon atoms only.

The molecular connectivity index is a topological descriptor of molecular structure based on a count of skeletal atom groupings of a chemical compound [4,5]. For each atom of a molecule, a $\delta$ value is assigned as the difference between the number of valence electrons and the number of hydrogen atoms attached to that atom. The first-order connectivity index was calculated as $[1,6,7]$ :

$$
{ }^{1} \chi=\sum_{q=1}^{n}\left(\delta_{i} \delta_{j}\right)_{q}^{-0.5}
$$

Where $\delta_{i}$ and $\delta_{j}$ are the $\delta$ values of two adjacent atoms $I$ and $j$, respectively; and $n$ is the number of bonds in the molecule.

\section{Results and Discussion}

As presented in Table 2, $K_{f g}$ was found to correlate best with the molecular weight of organic compounds $(r=0.832, p<0.05)$. Therefore, the molecular weight can be a good basis to understand the partition between SPME/PDMS and organic compounds in the gas. Table 3 indicates that $K_{f g}$ was positively correlate to the $\log K_{\text {ow }}(r=0.550, p<0.05)$ and the molecular connectivity index $(r=0.581, p<0.05)$ of organic compounds. Therefore, the 
partition of organic compounds between PDMS and the gaseous matrix was dependent on their polarity and structure of molecular.

Table 3 Correlation analysis of $K_{f g}$ for the training set

\begin{tabular}{|c|c|}
\hline Descriptor & Correlation \\
\hline$M_{\mathrm{W}}$ & $\log K_{f g}=-0.052+0.029 M_{\mathrm{W}}(r=0.832, p<0.05)$ \\
\hline${ }^{1} \chi$ & $\log K_{f g}=1.139+0.594^{1} \chi(r=0.581, p<0.05)$ \\
\hline $\log K_{\mathrm{ow}}$ & $\log K_{f g}=1.297+0.539 \log K_{\mathrm{ow}}(r=0.550, p<0.05)$ \\
\hline$\Phi$ & $\log K_{f g}=3.673-0.078 \Phi(r=-0.085, p>0.05)$ \\
\hline$W S$ & $\log K_{f g}=3.569-0.0001 W S(r=-0.257, p<0.05)$ \\
\hline
\end{tabular}

Due to the polarity of compounds is affected by hydrogen bond, molecular structure and symmetry, we grouped $K_{f g}$ into alkane and aromatic hydrocarbons. It was found that the correlation between molecular descriptors and $K_{f g}$ was better. The $K_{f g}$ of alkane and aromatic hydrocarbon was well correlated with ${ }^{1} \chi(r=0.609, r=0.961), \Phi(r=0.614, r=0.981)$ and $M_{\mathrm{W}}(r=0.615, r=0.981)$.

The best predictive model for $K_{f g}$ was obtained through the multiple regression analysis. Based on the stepwise regression with $95 \%$ confidence limits, the multiple regression analyse of $K_{f g}$ is presented as Eq. (3).

$$
\log K_{f g}=0.117-0.353 \Phi+0.034 M_{W}
$$

As shown in Fig. 1, there was a good agreement $(r=0.908)$ between the experimental $K_{f g}$ and those determined by Eq. (3), and there was no significant difference with a statistical analysis of the t-test $(p<0.05)$. Therefore, the predictive model of Eq. (3) can be employed to estimate $K_{f g}$ for SPME/PDMS to extract the analyte from the gaseous sample.

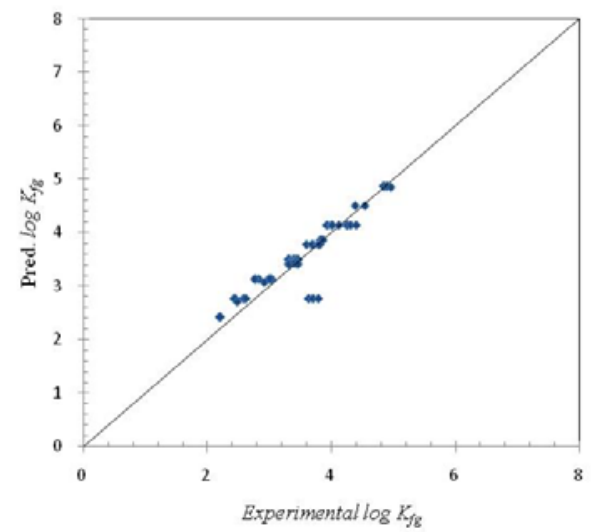

Fig. 1 Comparison of $K_{f g}$ between the experimental data and the predictive model. 


\section{Conclusion}

According to the single-parameter model analyses, the PDMS-gas partition coefficients were proportional to the molecular weight, molecular connectivity index, and octanol-water partition coefficient of organic compounds, while an inversely proportional trend was observed between $K_{f g}$, and polarizability as well as the water solubility. $K_{f g}$ was dependent on the molecular weight of the organic compounds most. Based on the results of multiple regression analyses, a correlation for $K_{f g}$ was developed using the molecular weight and the polarizability, indicating a good agreement $(r=0.908)$ between the experimental data and the predicted $K_{f g}$. The empirical model developed in this work is more versatile than current available correlations. In order to broaden the predictive abilities, however, future work should be conducted to calculate $K_{f g}$ using the empirical model for a variety of organic compounds.

\section{References}

1. Pawliszyn, J.,"Solid Phase Microextraction - Theory and Practice”, 1997, Wiley-VCH, New York.

2. Nirmalakhandan, N.N.;Speece, R.E. QSAR model for predicting Henry's constant. Environ. Sci. Technol.1988, 22, 1349-1357.

3. Horvath, A.L. Halogenated Hydrocarbons; Dekker: New York, NY, USA, 1982.

4. Kier, L.B.; Hall, L.H. Molecular Connectivity in Structure-Activity Analysis; Wiley: Chichester, UK, 1986.

5. Randic, M. On characterization of molecular branching. J. Am. Chem. Soc. 1975, 97, $6609-6615$.

6. Nirmalakhandan, N.N.;Speece, R.E. QSAR model for predicting Henry's constant. Environ. Sci. Technol.1988, 22, 1349-1357.

7. Zhao, H.; Zhang, Q.; Chen, J.;Xue, Z.; Liang, X. Prediction of octanol-air partition coefficients of semivolatile organic compounds based on molecular connectivity index. Chemosphere2005, 59, 1421-1426. 